\title{
Molecular jet emission and a spectroscopic survey of S235AB
}

\author{
R. A. Burns ${ }^{1}$, T. Handa ${ }^{1}$, T. Hirota ${ }^{2,3}$, K. Motogi ${ }^{4}$, H. Imai $^{1}$, and T. Omodaka ${ }^{1}$ \\ ${ }^{1}$ Graduate School of Science and Engineering, Kagoshima University, 1-21-35 Kôrimoto, 890-0065 Kagoshima, Japan \\ e-mail: RossBurns88@Googlemail.com \\ 2 Mizusawa VLBI Observatory, National Astronomical Observatory of Japan, Osawa 2-21-1, Mitaka, 181-8588 Tokyo, Japan \\ 3 Department of Astronomical Sciences, SOKENDAI (Graduate University for Advanced Studies), Osawa 2-21-1, Mitaka, \\ 181-8588 Tokyo, Japan \\ 4 Mizusawa VLBI Observatory, National Astronomical Observatory of Japan, 2-12 Hoshi-ga-oka, Mizusawa-ku, Oshu-shi, \\ 023-0861 Iwate, Japan
}

Received 24 August 2015 / Accepted 12 December 2015

\begin{abstract}
Context. The S235AB star forming region houses a massive young stellar object which has recently been reported to exhibit possible evidence of jet rotation - an illusive yet crucial component of disk aided star formation theories.

Aims. We confirm the presence of a molecular counterpart to the jet and we also study the molecular environment in S235AB.

Methods. We search for velocity wings in the line emission of thermal $\mathrm{SiO}(J=2-1, v=0)$, a tracer of shocked gas, which would indicate the presence of jet activity. Utilising other lines detected in our survey we use the relative intensities of intra species transitions, isotopes, and hyperfine transitions to derive opacities, temperatures, column densities, and abundances of various molecular species in $\mathrm{S} 235 \mathrm{AB}$.

Results. The $\mathrm{SiO}(J=2-1, v=0)$ emission exhibits velocity wings of up to $75 \mathrm{~km} \mathrm{~s}^{-1}$ above and below the velocity of the star, indicating the presence of a jet. The molecular environment describes an evolutionary stage resembling a hot molecular core.
\end{abstract}

Key words. stars: massive - ISM: jets and outflows

\section{Introduction}

The target of our study, $\mathrm{S} 235 \mathrm{AB}$, is a star forming region that houses a massive young stellar object (MYSO) called S235ABMIR, which was recently reported to exhibit fast $\left(\sim 50 \mathrm{~km} \mathrm{~s}^{-1}\right)$ jet-tracing water masers with a signature of rotation (Burns et al. 2015). The confirmation of a rotating jet in an MYSO would have a great impact on the theories of star formation as it would provide a method of removing angular momentum from the inner disk of the system and enabling accretion, while also corroborating a magneto-centrifugal launching mechanism for jets from massive young stars (see Konigl \& Pudritz 2000).

S235AB-MIR is known to have slow molecular outflows (Felli et al. 2004); however, aside from the fast water maser velocities, there is no evidence in the literature of a fast molecular jet in S235AB-MIR. In Felli et al. (2004) the authors claimed to have found a continuum jet aligned with the water maser jet; however, they later retracted the claim in Felli et al. (2006). As a follow-up to the maser jet results of Burns et al. (2015) the primary aim of our spectral line survey was to find the molecular counterpart to the water maser jet in S235AB, which should be readily identifiable from doppler broadened line "wings" seen in the thermal gas jet tracer $\operatorname{SiO}(J=2-1, v=0)$ (e.g. López-Sepulcre et al. 2011). The secondary aim of our observations was to further investigate the physical gas conditions in S235AB via basic astrochemical study using auxiliary spectral lines.

$\mathrm{S} 235 \mathrm{AB}$ is known to have a dense molecular core centered on S235AB-MIR and molecular outflows mapped in
$\mathrm{HCO}^{+}(1-0)$ and $\mathrm{C}^{34} \mathrm{~S}(5-4)$ (Felli et al. 2004), CS (7-6) (Wu et al. 2010), and ${ }^{13} \mathrm{CO}$ (Felli et al. 1997). The Spitzer colours indicate that S235AB-MIR has a mass of $11 M_{\odot}$, making it the only MYSO in the region (Dewangan \& Anandarao 2011), and non-detection of centimetre emission in S235AB attests to its youth (Tofani et al. 1995; Felli et al. 2006).

\section{Observations and data reduction}

We conducted molecular line observations of S235AB in March and April of 2015 with the $45 \mathrm{~m}$ radio telescope of the Nobeyama Radio Observatory (NRO), a branch of the National Astronomical Observatory of Japan.

We observed in two-sideband mode using the TZ receiver and we observed horizontal and vertical linear polarisations simultaneously. The upper sideband (USB) and lower sideband (LSB) central frequencies were 85.85 and $97.85 \mathrm{GHz}$, respectively, with band widths of $4 \mathrm{GHz}$ each. The beamsize was about $18^{\prime \prime}$. The SAM45 spectrometer provided 16 independent frequency arrays ( 8 per polarisation), which we organised to provide continuous coverage of a wide frequency range, and to include lines of particular interest. The frequency resolution was $244.14 \mathrm{kHz}$, providing a bandwidth of $1000 \mathrm{MHz}$ per array.

Observing coordinates were centred on the position of S235AB-MIR at $(\alpha, \delta)_{\mathrm{J} 2000.0}=\left(05^{\mathrm{h}} 40^{\mathrm{m}} 53.384+35^{\circ} 41^{\prime} 48^{\prime \prime} .447\right)$. Sky-level subtraction was performed using a region of empty sky at coordinates $(\alpha, \delta)_{\mathrm{J} 2000.0}=\left(05^{\mathrm{h}} 40^{\mathrm{m}} 58^{\mathrm{s}} .30+35^{\circ} 41^{\prime} 48^{\prime \prime} .^{\prime} 60\right)$. Absolute flux calibration was performed using the chopper wheel method, empty sky, and hot load. Pointing accuracy 
Table 1. Summary of detected molecular lines and their measured parameters.

\begin{tabular}{|c|c|c|c|c|c|c|}
\hline $\begin{array}{l}\text { Molecular } \\
\text { formula }\end{array}$ & Transition & $\begin{array}{l}T_{\mathrm{A}}^{*} \\
{[\mathrm{~K}]}\end{array}$ & $\begin{array}{c}\text { Obs frequency } \\
{[\mathrm{MHz}]}\end{array}$ & $\begin{array}{c}\Delta v \\
{[\mathrm{MHz}]}\end{array}$ & $\begin{array}{c}v_{\text {peak }} \\
{\left[\mathrm{km} \mathrm{s}^{-1}\right]}\end{array}$ & $\begin{array}{l}\mathrm{rms} \\
{[\mathrm{K}]}\end{array}$ \\
\hline $\mathrm{CH}_{3} \mathrm{OH}$ & $2(1,2)-1(1,1) \mathrm{A}++$ & 0.230 & 95914.406 & 0.819 & -17.22 & 0.012 \\
\hline $\mathrm{C}^{34} \mathrm{~S}$ & $2-1$ & 1.037 & 96413.016 & 0.852 & -17.13 & 0.008 \\
\hline $\mathrm{CH}_{3} \mathrm{OH}$ & $2(-1,2)-1(-1,1) \mathrm{E}$ & 0.962 & 96739.445 & 0.778 & -17.18 & 0.006 \\
\hline $\mathrm{CH}_{3} \mathrm{OH}$ & $2(0,2)-1(0,1) \mathrm{A}++$ & 1.330 & 96741.422 & 0.778 & -17.14 & 0.012 \\
\hline $\mathrm{CH}_{3} \mathrm{OH}$ & $2(0,2)-1(0,1) \mathrm{E}$ & 0.461 & 96744.617 & 0.836 & -17.21 & 0.016 \\
\hline $\mathrm{CH}_{3} \mathrm{OH}$ & $2(1,1)-1(1,0) \mathrm{E}$ & 0.210 & 96755.602 & 0.770 & -17.29 & 0.007 \\
\hline $\mathrm{C}^{33} \mathrm{~S}$ & $2-13 / 2-3 / 2$ & 0.030 & 97169.438 & 0.811 & -16.96 & 0.002 \\
\hline $\mathrm{C}^{33} \mathrm{~S}$ & $2-1 \quad 1 / 2-1 / 2$ & 0.151 & 97171.828 & 0.811 & -16.96 & 0.002 \\
\hline$C^{33} S$ & $2-13 / 2-1 / 2$ & 0.038 & 97175.188 & 1.155 & -16.74 & 0.003 \\
\hline OCS & $8-7$ & 0.048 & 97301.203 & 0.786 & -16.98 & 0.005 \\
\hline $\mathrm{CH}_{3} \mathrm{OH}$ & $2(1,1)-1(1,0) \mathrm{A}-$ & 0.191 & 97582.859 & 0.803 & -17.16 & 0.005 \\
\hline${ }^{34} \mathrm{SO}$ & $N, J=2,3-1,2$ & 0.094 & 97715.461 & 0.868 & -17.18 & 0.002 \\
\hline $\mathrm{CS}$ & $2-1$ & 3.639 & 97981.016 & 2.146 & -17.19 & 0.019 \\
\hline $\mathrm{CH}_{3} \mathrm{CHO}$ & $5(1,4)-4(1,3) \mathrm{E}$ & 0.030 & 98863.484 & 1.262 & -17.16 & 0.004 \\
\hline SO & $N, J=2,3-1,2$ & 1.531 & 99299.953 & 0.901 & -17.14 & 0.013 \\
\hline $\mathrm{CH}_{3} \mathrm{OH}$ & $5(-1,5)-4(0,4) E$ & 2.471 & 84521.188 & 3.170 & -16.94 & 0.020 \\
\hline OCS & $7-6$ & 0.075 & 85139.250 & 0.606 & -17.51 & 0.004 \\
\hline $\mathrm{HC}^{18} \mathrm{O}^{+}$ & $1-0$ & 0.035 & 85162.375 & 0.959 & -17.53 & 0.005 \\
\hline c- $\mathrm{C}_{3} \mathrm{H}_{2}$ & $2(1,2)-1(0,1)$ & 0.093 & 85338.969 & 0.614 & -17.22 & 0.005 \\
\hline $\mathrm{HCS}^{+}$ & $2-1$ & 0.129 & 85347.859 & 0.721 & -16.96 & 0.004 \\
\hline $\mathrm{CH}_{3} \mathrm{CCH}$ & $5(2)-4(2)$ & 0.034 & 85450.820 & 0.311 & -17.19 & 0.004 \\
\hline $\mathrm{CH}_{3} \mathrm{CCH}$ & $5(1)-4(1)$ & 0.058 & 85455.727 & 0.688 & -17.22 & 0.003 \\
\hline $\mathrm{CH}_{3} \mathrm{CCH}$ & $5(0)-4(0)$ & 0.085 & 85457.219 & 0.287 & -16.72 & 0.001 \\
\hline $\mathrm{HC}^{15} \mathrm{~N}$ & $1-0$ & 0.157 & 86054.992 & 0.795 & -17.09 & 0.005 \\
\hline SO & $N, J=2,2-1,1$ & 0.376 & 86093.984 & 0.721 & -17.00 & 0.005 \\
\hline $\mathrm{H}^{13} \mathrm{CN}$ & $1-0 F=1-1$ & 0.322 & 86338.750 & 0.786 & -17.05 & 0.005 \\
\hline $\mathrm{H}^{13} \mathrm{CN}$ & $1-0 F=2-1$ & 0.593 & 86340.180 & 0.811 & -17.01 & 0.004 \\
\hline $\mathrm{H}^{13} \mathrm{CN}$ & $1-0 F=0-1$ & 0.129 & 86342.242 & 0.688 & -16.95 & 0.004 \\
\hline $\mathrm{HCO}$ & $1(0,1)-0(0,0) 3 / 2-1 / 2 F=2-1$ & 0.044 & 86670.648 & 0.664 & -16.40 & 0.005 \\
\hline $\mathrm{H}^{13} \mathrm{CO}^{+}$ & $1-0$ & 0.282 & 86754.352 & 0.746 & -17.22 & 0.006 \\
\hline $\mathrm{SiO}$ & $2-1 v=0$ & 0.056 & 86847.023 & 1.901 & -17.04 & 0.001 \\
\hline $\mathrm{HN}^{13} \mathrm{C}$ & $1-0 F=2-1$ & 0.124 & 87090.812 & 0.705 & -16.84 & 0.005 \\
\hline $\mathrm{C}_{2} \mathrm{H}$ & $1-03 / 2-1 / 2 F=1-1$ & 0.137 & 87284.180 & 0.352 & -17.08 & 0.003 \\
\hline $\mathrm{C}_{2} \mathrm{H}$ & $1-03 / 2-1 / 2 F=2-1$ & 0.890 & 87316.969 & 0.770 & -17.15 & 0.008 \\
\hline $\mathrm{C}_{2} \mathrm{H}$ & $1-03 / 2-1 / 2 F=1-0$ & 0.479 & 87328.641 & 0.754 & -17.06 & 0.006 \\
\hline $\mathrm{C}_{2} \mathrm{H}$ & $1-01 / 2-1 / 2 F=1-1$ & 0.637 & 87402.102 & 0.778 & -17.34 & 0.008 \\
\hline $\mathrm{C}_{2} \mathrm{H}$ & $1-01 / 2-1 / 2 F=0-1$ & 0.296 & 87407.227 & 0.696 & -17.21 & 0.010 \\
\hline $\mathrm{C}_{2} \mathrm{H}$ & $1-01 / 2-1 / 2 F=1-0$ & 0.149 & 87446.523 & 0.819 & -17.03 & 0.008 \\
\hline
\end{tabular}

was checked using a nearby $\mathrm{SiO}$ maser source, RU Aur, every $\sim 1-2 \mathrm{~h}$.

During the March observations the atmospheric conditions were good with a typical temperature of $T_{\text {sys }} \approx 140 \mathrm{~K}$; however, strong wind conditions resulted in poor pointing accuracy and caused much of the on-source scans to suffer from beam warping and pointing offsets. Only $1 \mathrm{~h}$ of observing session provided usable data. During the April observations wind conditions were much better, providing good pointing accuracy (usually better than $3^{\prime \prime}$ ) and $T_{\text {sys }}$ was between 250 and $300 \mathrm{~K}$. Data from the full four-hour observing session was used.

To detect as many molecular lines as possible we alternated between two frequency array setups, each of which covered different frequency ranges. The only frequency array common to both setups was that containing the $\mathrm{SiO}$ line, which was our transition of highest priority. Subsequently, the frequency array containing the $\mathrm{SiO}$ line was observed for the full observing time, while other frequency arrays were observed for roughly half of this time.

Data reduction was performed using the NEWSTAR software developed by the NRO. To reduce the aforementioned effect of the wind in the March observations, we inspected all scans individually. Scans with bad pointing were determined by the failure in detecting $\mathrm{C}^{34} \mathrm{~S}$, a rarefied gas whose region of peak emission is known to be compact from the maps of Felli et al. (2004; their Fig. 9). This greatly improved the quality of integrated spectra.

After flagging bad scans, second-order polynomial baselines were fit to individual scans. Finally, all scans were integrated and polarisations were merged. Good scans on the target integrated to a total of $1 \mathrm{~h}$ for the March data and $2 \mathrm{~h}$ for the April data. The achieved sensitivity was typically better than $0.006 \mathrm{~K}$ for most frequency arrays, and reached $0.0025 \mathrm{~K}$ for the array containing the $\mathrm{SiO}$ emission. The antenna gain was roughly $4.4 \mathrm{Jy} / \mathrm{K}$. 


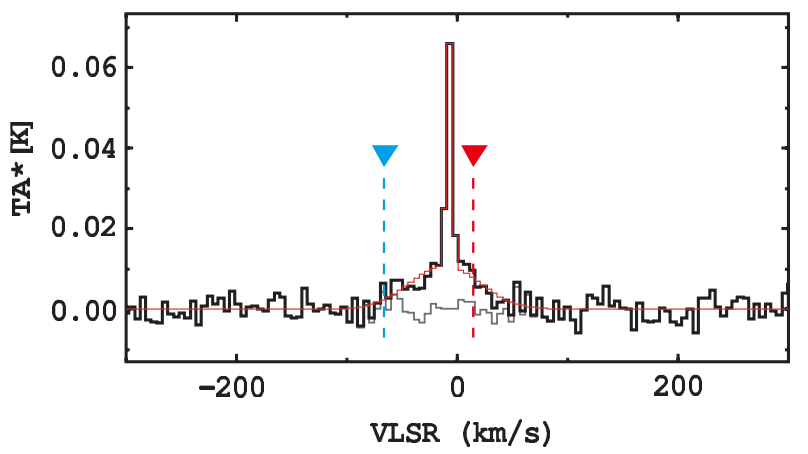

Fig. 1. Four-channel binned spectrum of the $\mathrm{SiO}(2-1)$ emission in S235AB. Black lines show the observed data while the combined two-Gaussian model and its residuals are shown in red and grey, respectively. Blue and red triangles indicate extremum velocities of the $\mathrm{H}_{2} \mathrm{O}$ masers of Burns et al. (2015).

\section{Results}

Gaussian profiles were fit to spectral lines detected above 5 times rms the noise. The detections and line parameters are listed in Table 1. Detected molecular transitions were identified by cross-referencing with the Cologne Database for Molecular Spectroscopy (CDMS) catalogue (Müller et al. 2005).

\subsection{SiO - jet tracer}

Our survey detected the common jet tracer transition line of $\mathrm{SiO}$ (2-1). In low-temperature environments $\mathrm{SiO}$ molecules are confined to dust grains, resulting in a low gas abundance. In a shocked gas environment these molecules are released from dust grains and become abundant in the gas phase (Martin-Pintado et al. 1992). As such $\mathrm{SiO}$ is a useful tracer of shocked gas, and velocity wings in its spectrum indicate the presence of jets and outflows (López-Sepulcre et al. 2011). The spectrum of $\mathrm{SiO}$ (2-1) gas in $\mathrm{S} 235 \mathrm{AB}$ is displayed in Fig. 1, showing wide velocity wings.

\subsection{Rotation temperature}

We detected multiple rotational transitions of sulphur monoxide (SO) such that it was possible to estimate its rotation temperature, $T_{\text {rot }}$, by using the "population diagram" method detailed in Goldsmith \& Langer (1999). Emission was optically thin and the derived rotation temperature was $T_{\text {rot }}=26 \pm 4 \mathrm{~K}$, which is similar to the value obtained by Felli et al. (2004) of $~ 30 \mathrm{~K}$ using transitions of $\mathrm{CH}_{3} \mathrm{CN}(5-4)$.

We also measured the rotation temperature of methanol using transitions of $\mathrm{CH}_{3} \mathrm{OH}(2-1)$, using lines of both E- and A-species. The population diagram is shown in Fig. 2, the slope gives $T_{\text {rot }}=10 \pm 1 \mathrm{~K}$ and the gas is optically thin. The line fit ignores the $\mathrm{CH}_{3} \mathrm{OH}(5-4) \mathrm{E}$ transition at $E_{\text {upper }}=40 \mathrm{~K}$, which we discuss below. Methanol in this frequency regime is thought to be subthermally excited (Menten et al. 1988). Our estimate can therefore be considered the lower limit of the kinematic temperature of the methanol cloud.

Multiple transitions of OCS were detected, but were too weak to attain a meaningful temperature estimate.

\subsection{Detection of a class I methanol maser}

The $\mathrm{CH}_{3} \mathrm{OH}$ (5-4) E transition (84.521188 GHz) deviates from its expected excitation conditions, as can be seen in the

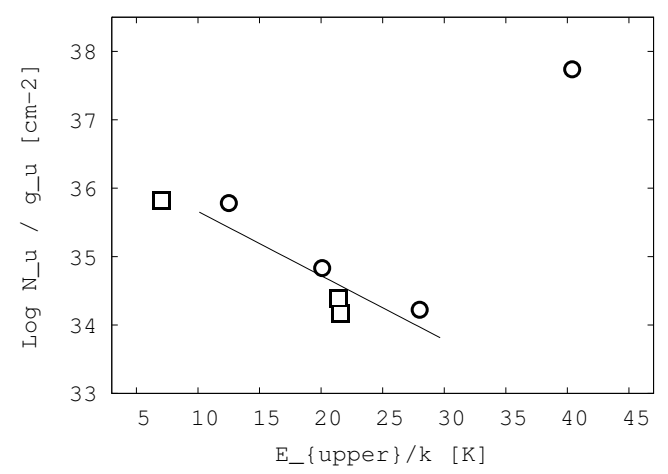

Fig. 2. Population diagram of $\mathrm{CH}_{3} \mathrm{OH}$ in $\mathrm{S} 235 \mathrm{AB}$. E- and A-species are indicated by circles and squares, respectively.

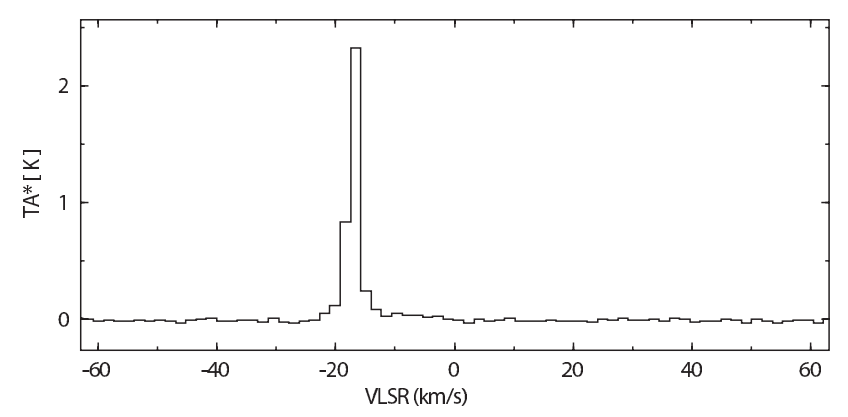

Fig. 3. Class I methanol maser emission spectrum from the $\mathrm{CH}_{3} \mathrm{OH}$ (5-4) E transition at $84.521188 \mathrm{GHz}$.

population diagram. This transition is known to exhibit maser behaviour (Menten et al. 1988); therefore, it seems that we have detected maser emission at this transition in S235AB (Fig. 3). The full line width of the emission is $11 \mathrm{~km} \mathrm{~s}^{-1}$, which is far too large to emanate from a single maser feature and thus indicates a likely blending of multiple features. The central velocity of this class I methanol maser is close to that of the molecular core, suggesting that it is not directly tracing the jet/outflow system and could instead be produced in the dense ambient gas somewhere near the outflow.

\subsection{Optical depth using hyperfine emission lines}

Intensity ratios of the main line $(F=2-1)$ to the hyperfine satellite line $(F=1-0)$ of the $\mathrm{C}_{2} \mathrm{H}$ molecule can be used to derive the gas optical depth via

Line intensity ratio $=\frac{1-\mathrm{e}^{-\tau}}{1-\mathrm{e}^{-a \tau}}$,

where $a$ is the intrinsic line intensity ratio determined by laboratory experiment. For $\mathrm{C}_{2} \mathrm{H}(F=1-0):(F=2-1), a=0.5$ in the optically thin limit (Tucker et al. 1974). Therefore, using Eq. (1) our observed line intensity ratio of $T_{\mathrm{A}}(1-0) / T_{\mathrm{A}}(2-1)=0.55$ gives $\tau_{\mathrm{C}_{2 \mathrm{H}}}=0.39$. This value is typical of young, massive, star forming clumps (Sakai et al. 2010).

For optically thin $\mathrm{H}^{13} \mathrm{CN}$ the intrinsic intensity ratios of the hyperfine transitions $(F=1-1):(F=2-1):(F=1-0)$ are $3: 5: 1$ (Irvine \& Schloerb 1984). Taking the transitions $(F=2-1):(F=$ $1-0)=5$, our observed intensity ratio of $T_{\mathrm{A}}(F=2-1) / T_{\mathrm{A}}(F=$ $1-0)=4.36$ gives $\tau_{\mathrm{H}^{13} \mathrm{CN}}=0.07$. 
Table 2. Summary of derived physical parameters.

\begin{tabular}{ccccc}
\hline \hline Molecule & $\tau$ & $\begin{array}{c}T_{\text {rot }} \\
{[\mathrm{K}]}\end{array}$ & $\begin{array}{c}N_{\text {Tot }} \\
{\left[\mathrm{cm}^{-2}\right]}\end{array}$ & $\begin{array}{c}\text { Molecular } \\
\text { abundance }\end{array}$ \\
\hline $\mathrm{C}_{2} \mathrm{H}$ & 0.39 & - & $3.50 \mathrm{E}+15$ & $3.5 \times 10^{-8}$ \\
$\mathrm{H}^{13} \mathrm{CN}$ & 0.07 & - & $5.80 \mathrm{E}+13$ & $5.7 \times 10^{-10}$ \\
$\mathrm{CS}$ & 5.1 & - & $6.63 \mathrm{E}+15$ & $6.6 \times 10^{-8}$ \\
$\mathrm{SO}$ & 0.9 & $26 \pm 4$ & $9.76 \mathrm{E}+14$ & $9.7 \times 10^{-9}$ \\
$\mathrm{CH}_{3} \mathrm{OH}$ & $\ll 1$ & $10 \pm 1$ & $7.85 \mathrm{E}+15$ & $7.8 \times 10^{-8}$ \\
\hline
\end{tabular}

\subsection{Optical depth using isotopic abundance}

We detected two isotopologues of two sulphur-bearing species: $\mathrm{C}^{34} \mathrm{~S}$ and $\mathrm{CS}$, and ${ }^{34} \mathrm{SO}$ and SO. We estimated the optical depths of CS and SO using the isotopologue line intensity ratios, as was done in Eq. (1), where $a$ is the intrinsic intensity ratio of ${ }^{32} \mathrm{~S} /{ }^{34} \mathrm{~S}=22.5$ (Kahane et al. 1988). Our measured intensity ratio of $T_{\mathrm{A}}(\mathrm{CS}) / T_{\mathrm{A}}\left(\mathrm{C}^{34} \mathrm{~S}\right)=4.9$ gives $\tau_{\mathrm{CS}}=5.1$, and our intensity ratio of $T_{\mathrm{A}}\left({ }^{34} \mathrm{SO}\right) / T_{\mathrm{A}}(\mathrm{SO})=15.2$ gives $\tau_{\mathrm{SO}}=0.9$.

\subsection{Column densities and abundances}

To estimate column densities from individual molecular species, we used the prescription outlined in Goldsmith \& Langer (1999) for species with measurable optical depths (see Sects. 3.4 and 3.5) except for the column density of $\mathrm{CH}_{3} \mathrm{OH}$, which was obtained from the population diagram. For other molecules the statistical weights, upper energy levels, partition functions, and Einstein coefficients for individual transitions were sourced from the Leiden Atomic and Molecular Database (LAMBDA; Schöier et al. 2005) and CDMS catalogues with an assumed excitation temperature of $18.75 \mathrm{~K}$ with the exception of $\mathrm{SO}$, for which we used the measured rotation temperature (see Sect. 3.2).

Saito et al. (2007) estimated the molecular hydrogen column density of the molecular core in S235AB to be $10.1 \times 10^{22} \mathrm{~cm}^{-2}$ based on their $\mathrm{C}^{18} \mathrm{O}$ observations at comparable angular resolution. By comparing their results with the column densities measured in this work we estimated molecular abundances. These, alongside the aforementioned physical gas parameters, are summarised in Table 2.

\section{Discussion and conclusions}

Observations of S235AB by Sun et al. (2012) detected SiO emission at a peak brightness consistent with our observations; however, velocity wings were not detectable at the $0.017 \mathrm{~K} \mathrm{rms}$ sensitivity of their observations. Our $\mathrm{SiO}$ spectrum (Fig. 1) exhibits wide velocity wings reaching up to $75 \mathrm{~km} \mathrm{~s}^{-1}$ from the core velocity. Our deeper observations confirm the presence of a fast molecular jet in S235AB. Discovery of a high-velocity jet in this source is of particular significance in light of the recent water maser results of Burns et al. (2015). Those authors find both blueshifted and redshifted high-velocity $\left(\sim 50 \mathrm{~km} \mathrm{~s}^{-1}\right.$ from the core velocity) maser features in a bipolar configuration matching the alignment of the slow $\left(<5 \mathrm{~km} \mathrm{~s}^{-1}\right.$ from the core velocity) NNW-SSE molecular outflow seen in $\mathrm{HCO}^{+}$and $\mathrm{C}^{34} \mathrm{~S}$, reported by Felli et al. (2004).

The velocity of the $\mathrm{SiO}$ gas $\left(\sim 75 \mathrm{~km} \mathrm{~s}^{-1}\right)$ is much faster than that of the molecular outflow in S235AB making it likely that the $\mathrm{SiO}$ emission traces the primary molecular jet. Furthermore, as can be seen in Fig. 1, the $\mathrm{SiO}$ jet gas and water masers have similar terminal velocities, which suggests likely association, although this should be confirmed with mapping observation.

Molecular outflows are thought to be produced as linear momentum from a protostellar jet is transferred into ambient gas around to the protostar. This gas becomes entrained at a slower velocity and at larger radii from the inner jet. The fast $\mathrm{SiO}$ gas in S235AB-MIR therefore indicates the presence of an entraining jet, which is the driving source for the water masers and molecular outflows seen in this region.

Burns et al. (2015) report evidence of possible jet rotation in their maser observations. Our confirmation of a molecular jet in S235AB-MIR introduces the possibility of investigating the spatial orientation of the jet via mapping observations, which may unearth further evidence of jet rotation.

Our survey investigated the physical properties of numerous molecular species in $\mathrm{S} 235 \mathrm{AB}$, which revealed a cold, dense gas environment with high opacity in several tracers typical of the young cores in which MYSOs are embedded. Our survey results provide information on strengths and line widths of various gas tracers, which will be useful for further follow-up observations.

We detected maser emission in the $\mathrm{CH}_{3} \mathrm{OH}(5-4) \mathrm{E}$ transition at $84.521188 \mathrm{GHz}$ emanating from an ensemble of blended features. With regard to thermal $\mathrm{CH}_{3} \mathrm{OH}$ emission at $97 \mathrm{GHz}$, we measured a low rotation temperature for the methanol gas $(\sim 10 \mathrm{~K})$ derived from the population diagram (Fig. 3). In this respect $\mathrm{S} 235 \mathrm{AB}$ conforms to the trend reported by Minier \& Booth (2002) who find low rotation temperatures to be a systematic feature among their sample of massive star forming regions. They postulate that the $97 \mathrm{GHz}$ methanol gas may trace cooler gas at the outer parts of the methanol cloud.

With regard to molecular abundances, we compare our results to Gerner et al. (2014) who have investigated a sample of 59 massive star forming regions categorised into different stages of evolution which, in order of ascending evolutionary stage, are infrared dark clouds, high-mass protostellar objects, hot molecular cores, and ultra compact $\mathrm{H}_{\mathrm{II}}$ regions. A comparison with their results was possible for $\mathrm{C}_{2} \mathrm{H}, \mathrm{SO}, \mathrm{CS}$, and $\mathrm{CH}_{3} \mathrm{OH}$, which are common to both our work and theirs. For the cases of $\mathrm{C}_{2} \mathrm{H}, \mathrm{SO}$, and $\mathrm{CH}_{3} \mathrm{OH}$ our estimated abundances in $\mathrm{S} 235 \mathrm{AB}$ are consistent with the hot molecular core category of Gerner et al. (2014; see their Fig. 3), while CS matches somewhere between the hot molecular core and high-mass protostellar object categories (see their Fig. 5). The abundance of $\mathrm{H}^{13} \mathrm{CN}$ in $\mathrm{S} 235 \mathrm{AB}$ is similar to those seen in another five well-studied massive star forming regions, the survey of which was reported in Zinchenko et al. (2009).

To conclude, the molecular abundances in S235AB are typical of a young massive star forming region. Its evolutionary stage close to that of a hot molecular core (this work and Felli et al. 2004) and preceding the formation of a HII region, as is indicated by the lack of centimetre emission (Tofani et al. 1995; Felli et al. 2006). Nonetheless, it is a very active region as can be seen by the presence of slow molecular outflows (Felli et al. 2004), a maser jet (Burns et al. 2015), and a fast molecular jet.

Acknowledgements. We would like to thank the staff at NRO for their generous support. We would also like to thank Takano Shuro for interesting discussions during the observing periods. R.B. would like to acknowledge the Ministry of Education, Culture, Sports, Science and Technology (MEXT), Japan, for financial support under the Monbukagakusho scholarship. T. Hirota is supported by the MEXT/JSPS KAKENHI Grant Numbers 24684011, 25108005, and $15 \mathrm{H} 03646$. H.I. is also supported by the JSPS KAKENHI Grant Number 25610043. K.M. is supported by a Grant-in-Aid from the JSPS Fellows and JSPS KAKENHI Grant numbers 24-6525 and 15K17613. 
R. A. Burns et al.: Molecular jet emission and a spectroscopic survey of S235AB

\section{References}

Burns, R. A., Imai, H., Handa, T., et al. 2015, MNRAS, 453, 3163

Dewangan, L. K., \& Anandarao, B. G. 2011, MNRAS, 414, 1526

Felli, M., Testi, L., Valdettaro, R., \& Wang, J.-J. 1997, A\&A, 320, 594

Felli, M., Massi, F., Navarrini, A., et al. 2004, A\&A, 420, 553

Felli, M., Massi, F., Robberto, M., \& Cesaroni, R. 2006, A\&A, 453, 911

Gerner, T., Beuther, H., Semenov, D., et al. 2014, A\&A, 563, A97

Goldsmith, P. F., \& Langer, W. D. 1999, ApJ, 517, 209

Irvine, W. M., \& Schloerb, F. P. 1984, ApJ, 282, 516

Kahane, C., Gomez-Gonzalez, J., Cernicharo, J., \& Guelin, M. 1988, A\&A, 190, 167

Konigl, A., \& Pudritz, R. E. 2000, Protostars and Planets IV, 759

López-Sepulcre, A., Walmsley, C. M., Cesaroni, R., et al. 2011, A\&A, 526, L2

Martin-Pintado, J., Bachiller, R., \& Fuente, A. 1992, A\&A, 254, 315
Menten, K. M., Walmsley, C. M., Henkel, C., \& Wilson, T. L. 1988, A\&A, 198, 253

Minier, V., \& Booth, R. S. 2002, A\&A, 387, 179

Müller, H. S., Schlöder, F., Stutzki, J., \& Winnewisser, G. 2005, J. Mol. Struct., 742,215

Saito, H., Saito, M., Sunada, K., \& Yonekura, Y. 2007, ApJ, 659, 459

Sakai, T., Sakai, N., Hirota, T., \& Yamamoto, S. 2010, ApJ, 714, 1658 Schöier, F. L., van der Tak, F. F. S., van Dishoeck, E. F., \& Black, J. H. 2005 A\&A, 432, 369

Sun, Y., Xu, Y., \& Yang, J. 2012, Chin. Astron. Astrophys., 36, 238

Tofani, G., Felli, M., Taylor, G. B., \& Hunter, T. R. 1995, A\&AS, 112, 299

Tucker, K. D., Kutner, M. L., \& Thaddeus, P. 1974, ApJ, 193, L115

Wu, J., Evans, II, N. J., Shirley, Y. L., \& Knez, C. 2010, ApJS, 188, 313

Zinchenko, I., Caselli, P., \& Pirogov, L. 2009, MNRAS, 395, 2234 